\title{
A sub-Doppler resolution double resonance molecular beam infrared spectrometer operating at chemically relevant energies $(\sim 2 \mathrm{eV})$
}

\author{
H. K. Srivastava, A. Conjusteau, H. Mabuchi, ${ }^{a)}$ A. Callegari, ${ }^{\text {b) }}$ K. K. Lehmann, \\ and G. Scoles ${ }^{\mathrm{c})}$ \\ Department of Chemistry, Princeton University, Princeton, New Jersey 08544
}

(Received 22 March 2000; accepted for publication 19 July 2000)

\begin{abstract}
A molecular beam spectrometer capable of achieving sub-Doppler resolution at $2 \mathrm{eV}(\sim 18000$ $\mathrm{cm}^{-1}$ ) of vibrational excitation is described and its performance demonstrated using the $\mathrm{CH}$ stretch chromophore of HCN. Two high finesse resonant power-buildup cavities are used to excite the molecules using a sequential double resonance technique. A $v=0 \rightarrow 2$ transition is first saturated using a $1.5 \mu \mathrm{m}$ color center laser, whereupon a fraction of the molecules is further excited to the $v=6$ level using an amplitude modulated $\mathrm{Ti}_{\mathrm{Al}} \mathrm{Al}_{2} \mathrm{O}_{3}$ laser. The energy absorbed by the molecules is detected downstream of both excitation points by a cryogenically cooled bolometer using phase sensitive detection. A resolution of approximately $15 \mathrm{MHz}$ (i.e., three parts in $10^{8}$ ) is demonstrated by recording a rotational line in the $v=6$ manifold of HCN. Scan speeds of up to several $\mathrm{cm}^{-1} / \mathrm{h}$ were obtained, with signal-to-noise ratios in excess of 100 . The high signal-to-noise ratio and a dynamic range of $6 \times 10^{4}$ means that future experiments to study statistical intramolecular vibrational energy redistribution in small molecules and unimolecular isomerizations can be attempted. We would also like to point out that, with improved metrology in laser wavelengths, this instrument can also be used to provide improved secondary frequency standards based upon the rovibrational spectra of molecules. (C) 2000 American Institute of Physics.
\end{abstract}

[S0034-6748(00)05010-3]

\section{INTRODUCTION}

As the density of accessible states increases, eigenstateresolved spectroscopy becomes, of course, increasingly difficult, and eventually impossible when the average transition separation becomes smaller than the spectrometer resolution. The density of vibrational states can become too large either when the number of atoms or when the energy contents of the molecule become too large.

There are several reasons for pushing the limits at which highly vibrationally excited polyatomic molecules can be studied with eigenstate resolution. To begin with, it is interesting to investigate how (at least for $X-H$ stretches) the local mode description ${ }^{1-3}$ of vibrational excitation becomes, as expected, more prevalent over the normal mode picture. Additionally, important insights into unimolecular dynamics can be obtained from eigenstate resolved spectroscopy of molecules undergoing isomerization and/or bond breaking at chemically relevant energies. Finally, climbing higher in the vibrational manifold allows for the study of unimolecular dynamics in small molecules (which can be treated precisely with modern day calculation methods) in the region of the density of states where state mixing may occur.

Unimolecular dynamics has been an area of sustained

\footnotetext{
a) On leave from: Department of Physics, California Institute of Technology, Pasadena, CA 91125.

${ }^{b)}$ Present address: Laboratoire de Chimie Physique Moléculaire (LCPM), École Polytechnique Fédérale de Lausanne, CH-1015 Lausanne, Switzerland.

${ }^{c)}$ Author to whom correspondence should be addressed; electronic mail: gscoles@princeton.edu
}

interest in the chemical community for quite some time. ${ }^{4,5}$ Recent experimental and theoretical reviews ${ }^{6-9}$ have demonstrated the importance of understanding the nature of intramolecular vibrational energy redistribution (IVR) in fields such as chemical dynamics and laser selective chemistry. Recent frequency domain experiments ${ }^{10-29}$ have shown the efficacy of eigenstate resolved spectroscopy as a tool for the exploration of IVR behavior at "long" times. While a wide array of molecules have been investigated, the studies carried out so far on relatively large ( $>6$ atoms) molecules were done at modest levels of vibrational excitation-typically one or two quanta in the $\mathrm{CH}$ stretching coordinate. At these low levels of vibrational excitation, statistical behavior is achieved only for molecules with more than 10 atoms, which are not yet amenable to quasiexact force field calculations.

Higher energy studies have been carried out in the past, but in order to overcome the low transition dipole associated with overtone transitions, they typically have been done in long path length gas cells. As the width of a spectral feature Fourier transforms into the longest time scale of the dynamics being studied, the resulting (Doppler and pressure broadened) low resolution spectra yield little quantitative information beyond the very early time dynamics. Recently, Rizzo and Settle have developed a technique that allows the acquisition of medium resolution spectra at high energies, ${ }^{30}$ and have applied it to identify multiple time scales of vibrational relaxation in molecules such as methanol. ${ }^{31,32}$ However, the pulsed laser employed to do the overtone excitation limits the experimental resolution to $0.02 \mathrm{~cm}^{-1}(600 \mathrm{MHz})$, which imposes a long time upper bound of $250 \mathrm{ps.} \mathrm{In} \mathrm{contrast,} \mathrm{the}$ 
spectrometer described in this paper has a resolution of $0.0005 \mathrm{~cm}^{-1}(15 \mathrm{MHz})$, which means that IVR on time scales as long as $10 \mathrm{~ns}$ can be explored.

In this article, we describe a recently constructed optothermal infrared spectrometer capable of depositing in excess of $2 \mathrm{eV}$ of vibrational energy per molecule into a collimated molecular beam with an energy resolution of 3 parts in $10^{8}$. For instance, we achieve the excitation to the $v=6$ level of a $\mathrm{CH}$ stretch chromophore in two steps: first we saturate the $v=0 \rightarrow 2$ transition followed by a $v=2 \rightarrow 6$ excitation. This sequential double resonance technique has two major advantages. The first is that due to the anharmonicity of the $v$ $=2$ level, the transition dipole for the $2 \rightarrow 6$ step is a factor of 32 greater than that for the corresponding $0 \rightarrow 6$ transition. ${ }^{33}$ Since the intensity of a molecular transition scales with the square of the transition dipole, the double resonance technique yields an effective enhancement of $10^{3}$ over the single photon. The second advantage is that because all the $2 \rightarrow 6$ transitions originate from a single, assigned $v$ $=2$ level, the lines at the $v=6$ level can be assigned from a rather restricted number of possibilities. In addition, for molecules with a center of inversion, $g / u$ selection rules for a two photon transition mean that we probe states with no net change in symmetry. The only drawback of the double resonance technique as realized here (apart from the experimental complexity of maintaining two photon sources and ensuring that the same portion of the molecular beam is pumped by both lasers) is that, unless more complex modulation schemes are adopted, it is not background free, since the 2 $\rightarrow 6$ spectrum rides on top of a large dc $0 \rightarrow 2$ signal.

\section{THE EXPERIMENTAL APPARATUS}

The spectrometer consists of four major subsystems, each of which will be described in detail in the following pages: the molecular beam system, the $1.5 \mu \mathrm{m}$ laser system, the $0.8 \mu \mathrm{m}$ laser system, and the computer control system. The molecular beam and $1.5 \mu \mathrm{m}$ laser systems preexisted in the present setup, and required only minor modifications to adapt them to the apparatus described below. The $0.8 \mu \mathrm{m}$ laser system combined with its power buildup cavity, on the other hand, represents a "new technology" in the lab, and as such, offered the greatest challenges.

In brief, a molecular beam (typically a $1 \%$ mixture of the analyte seeded in helium) is formed and then is made to interact successively with light from the 1.5 and $0.8 \mu \mathrm{m}$ lasers within fiber-fed cavities, which are resonantly tuned with their respective laser sources. Owing to the long spontaneous infrared emission lifetimes, vibrational energy adsorbed by the molecules can be synchronously detected downstream of the excitation regions with a cryogenically cooled bolometer.

\section{A. Molecular beam system-overview}

A schematic of the molecular beam system used in this experiment is shown in Fig. 1. The instrument consists of two differentially pumped chambers, each pumped by an Edwards EO400 (7000 1/s) oil diffusion pump, backed by a single Edwards rotary/roots mechanical pump combination.

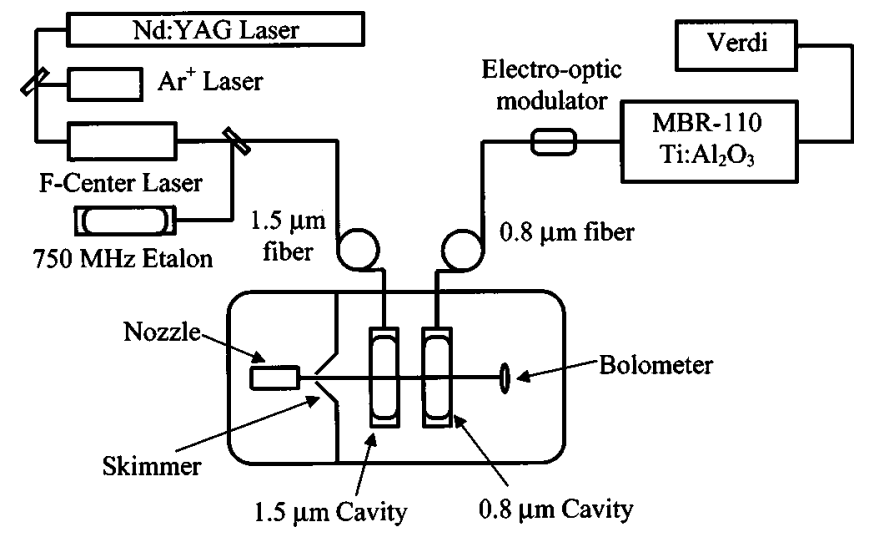

FIG. 1. Diagram of experimental apparatus.

The background pressures (nominal for air) in both chambers are on the order of $2 \times 10^{-7}$ Torr, and when the spectrometer is in operation, the pressure in the source chamber rises to approximately $1 \times 10^{-4}$ Torr and that of the detector chamber to $4 \times 10^{-7}$ Torr. The molecular beam system consists of three major subcomponents: a beam source, two resonant power buildup cavities, and a bolometric detector, each of which will be described in more detail.

\section{Molecular beam system-beam source}

The molecular beam is formed by expanding a mixture of $1 \% \mathrm{HCN}$ in $99 \%$ He through a $30 \mu \mathrm{m}$ diam nozzle, ${ }^{34}$ at a typical stagnation pressures of about 8 bar. The nozzle itself is mounted on a Motor Mike ${ }^{\text {TM }}$ (a trademark of Oriel Instruments) controlled three-axis translation stage in order to optimize the molecular flux through the fixed skimmer. The center line portion of the expansion is extracted by a $275 \mu \mathrm{m}$ conical skimmer located approximately $1 \mathrm{~cm}$ downstream of the nozzle. The skimmer isolates the high pressure source chamber from the low pressure detector chamber. After passing through the skimmer, the molecular beam is an essentially collisionless environment, with an average rotational temperature of $\sim 3 \mathrm{~K}$ (see Sec. III A).

After the molecular beam enters the detector chamber, it passes through the 1.5 and $0.8 \mu \mathrm{m}$ buildup cavities sequentially, located roughly 4 and $6.5 \mathrm{~cm}$ from the skimmer, respectively. The bolometer is located approximately $16 \mathrm{~cm}$ downstream of the skimmer, collimated by a $1 \mathrm{~mm}$ wide vertical slit located $5.5 \mathrm{~cm}$ upstream. With the given expansion parameters, the beam has an average translational velocity of about $1750 \mathrm{~m} / \mathrm{s}$, which implies a flight time from skimmer to detector of about $100 \mu \mathrm{s}$.

\section{Molecular beam system-buildup cavities}

Due to the small transition dipole associated with overtone transitions, the excitation of an appreciable percentage of the molecules in the molecular beam requires the use of high laser power densities. We achieve this goal by making the molecular beam and the lasers interact inside of a pair of high finesse resonant power buildup cavities (BUCs). The BUCs are essentially external resonators, and the laser power circulating inside them is "built up" to a higher value based upon their finesse and efficiency. 

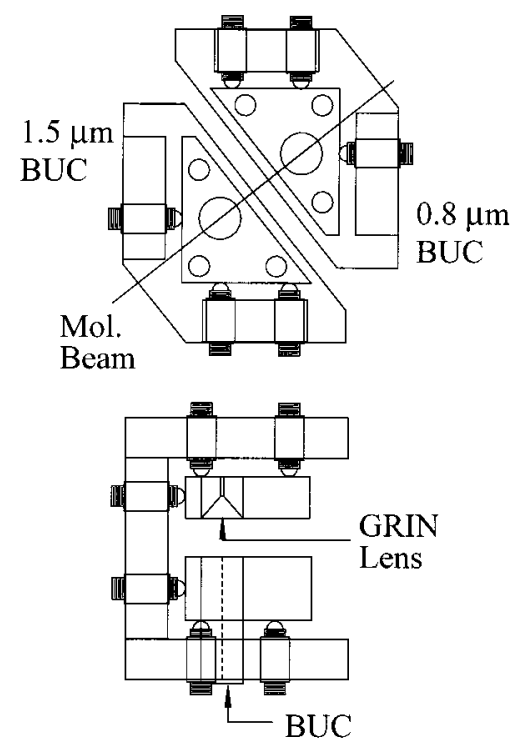

FIG. 2. Schematic of the buildup cavity mounts, top and side views (only the $1.5 \mu \mathrm{m}$ BUC is shown in the side view). The small circles in the top view are the vertical adjustment screws (visible in the side view) for the GRIN lens and BUC plates, while the large circle represents the GRIN lens and BUC themselves. In the side view, the portion of the mount in the plane of the page has been cut away to show the plates housing the GRIN lens and BUC. As shown in the top view, the molecular beam passes through the two BUCs in series, although the hole in the center of the cavity is not visible in the side view.

Both cavities were purchased from Newport, and are essentially the optical component of their SR-100 series spectrum analyzer. They consist of two high reflectivity mirrors $(R>0.9995)$ epoxied to a 1 in. long $(2.54 \mathrm{~cm})$ piezoelectric tube (PZT). The PZT has two holes custom drilled on an axis perpendicular to the axis of the PZT to allow passage of the molecular beam. Both cavities tune one free spectral range (6 $\mathrm{GHz}$ ) upon application of a modest voltage to the PZT $(<100 \mathrm{~V})$. The mirrors have radii of curvature of $30 \mathrm{~cm}$, and hence, the cavities are nonconfocal. A graded index (GRIN) lens is used to mode match the laser beam to the lowest order cavity mode. Both cavities are mounted vertically in a home built cavity holder which also supports the GRIN lenses and has 12 degrees of freedom adjustable with micrometer screws (see Fig. 2). When the cavities are properly mode matched, the peak heights of the higher order transverse modes are less than $5 \%$ of the peak height of the $\mathrm{TEM}_{00}$ mode.

In order to decouple the cavity alignment from laser beam pointing fluctuations and mechanical vibrations, optical fibers are used to couple the light into the BUCs. Typically $50 \%-70 \%$ of the available laser power is coupled into the optical fiber using a five-axis fiber positioner (Newport F-916 series). The laser light is brought into the machine through an FC bulk head connector, which mates the external fiber to an identically connectorized one inside the spectrometer. It has been experimentally determined that less than $10 \%$ of the coupled laser power is lost during transmission through the fibers and connectors.

The measured finesse of the BUCs at their central wavelengths are 6000 and 19000 for the 1.5 and $0.8 \mu \mathrm{m}$ cavities, respectively. The finesse of the cavities decreases away from the central wavelength, but remains greater than 5000 for the $1.5 \mu \mathrm{m}$ and 10000 for the $0.8 \mu \mathrm{m}$ cavity within $10 \%$ of the central wavelength. Based upon these values, and the measured efficiencies (the ratio of output power to input power, on resonance), the power buildup of the two cavities is calculated to be 900 and 4500 at the peak of their tuning curves. Over the complete tuning range of the cavities, the gains are greater than 100 and 1000, respectively. We have noted, however, that over time the mirrors become contaminated with diffusion pump oil, which decreases the finesse and increases the absorptive losses, resulting in a less than optimal power buildup. For this reason, a precise determination of the gain is not possible, but the departure from ideality is estimated to be less than a factor of 2 . The decreased finesse is marked by an increase in the transmission width of the cavity, which can be measured by sweeping the cavity with a slow triangular ramp. Previous experience with BUCs in our laboratory has indicated that the finesse of the cavities remains at a usable value for about $1 \mathrm{yr}$ under continuous operation. After this time the BUCs need to be extracted from the machine and cleaned before they can be reused. We have found that flowing warm nitrogen gas through the holes in the PZT for several days is sufficient to restore the finesse of the cavity to its performance specifications.

The beam waist of the $\mathrm{TEM}_{00}$ modes inside the BUCs are calculated to be 0.171 and $0.127 \mathrm{~mm}$ for the 1.5 and 0.8 $\mu \mathrm{m}$ cavities, respectively. With the calculated velocity of the molecular beam, this corresponds to a transit time broadening full width half maximum (FWHM) of $4 \mathrm{MHz}$ for the 1.5 $\mu \mathrm{m}$ cavity and $5 \mathrm{MHz}$ for the $0.8 \mu \mathrm{m}$ cavity. ${ }^{35}$

\section{Molecular beam system-bolometric detector}

The detector in this spectrometer is a composite-type germanium bolometer from Infrared Laboratory (unit No. 657). The bolometer is mounted on a copper cold finger, which is attached to the bottom of a liquid helium cryostat and surrounded by a liquid nitrogen cryostat. A rotary/roots combination is used to reduce the vapor pressure above the helium reservoir to about 1 Torr, which in turn reduces the temperature of the bath to approximately $1.6 \mathrm{~K}$. The low working temperature of the bolometer decreases microphonic noise, since below the $\lambda$ point $(2.18 \mathrm{~K})$ liquid helium behaves as a superfluid, and evaporates only from the surface instead of bubbling through the bulk. A copper cold shield attached to the liquid nitrogen cryostat envelops the bolometer and provides radiation shielding. The molecular beam reaches the bolometer through a $1 \mathrm{~mm}$ wide by $15 \mathrm{~mm}$ high vertical slit in the copper shield. The bolometer noise is 17 $\mathrm{nV} / \mathrm{Hz}^{1 / 2}$ and has a calculated noise equivalent power of 9 $\times 10^{-14} \mathrm{~W} / \mathrm{Hz}^{1 / 2}$. The bolometer response (specified to be $2.32 \times 10^{5} \mathrm{~V} / \mathrm{W}$ ) is essentially flat at low frequencies, with the $3 \mathrm{~dB}$ point occurring at about $600 \mathrm{~Hz}$. For the experiments reported here, synchronous detection is performed at around $310 \mathrm{~Hz}$.

\section{B. The $1.5 \mu \mathrm{m}$ laser system}

The $1.5 \mu \mathrm{m}$ laser system has been described before. ${ }^{36}$ Briefly, it consists of a Burleigh model FCL-220 $\mathrm{F}_{2 \mathrm{H}}^{+}$color 
center laser. $4 \mathrm{~W}$ of $1064 \mathrm{~nm}$ radiation from a Quantronix $416 \mathrm{Nd}$ :YAG laser are used to pump transitions of $\mathrm{F}_{2 \mathrm{H}}^{+}$defects in a hydroxide doped sodium chloride crystal. In addition, $10 \mathrm{~mW}$ of blue-green light (provided by an Omnichrome model $532 \mathrm{Ar}^{+}$laser operating on all lines) is required to electronically pump and reorient the absorptive dipole of the F centers. The F-center laser output is continuously tunable from 5700 to $6700 \mathrm{~cm}^{-1}$ at output powers greater than $100 \mathrm{~mW}$. The resulting free running linewidth of the color center laser is approximately $15 \mathrm{MHz}$. Power fluctuations of the Nd:YAG pump laser are believed to be the major contributor to the free running linewidth, since power fluctuations in the pump cause the temperature (and hence, index of refraction) of the laser crystal to change, which leads to fluctuations in the length of the laser cavity. The thermal tuning rate of the laser crystal is approximately 100 $\mathrm{kHz} / \mathrm{mW}^{37}$

In order to efficiently couple light into the BUC, the laser frequency must coincide with a cavity transmission peak to within a small fraction of the transmission linewidth of the BUC. For a finesse of 6000 and a $6 \mathrm{GHz}$ free spectral range, the spectral width of a cavity mode is $1 \mathrm{MHz}$. The needed improvement in the frequency stability of the color center laser was achieved by the usage of an intracavity $\mathrm{Li}: \mathrm{NbO}_{3}$ electro-optic crystal. An applied voltage across the electrodes of the crystal changes its index of refraction, which in turn changes the laser frequency. Modulating the voltage applied to the crystal with a small sine wave voltage $(\sim 75 \mathrm{kHz})$ causes the effective laser frequency to oscillate by a small amount $(\sim 50 \mathrm{kHz})$ around its average frequency. By synchronously phase detecting the modulated transmission through the BUC and feeding the error signal back into the electro-optic crystal, the frequency jitter of the laser has been reduced to about $100 \mathrm{kHz}{ }^{33}$ The integrated feedback signal is used to lock the BUC to the laser.

In a double resonance experiment, the F-center laser is made to stay on top of a narrow (15 MHz) $0 \rightarrow 2$ molecular beam transition. To accomplish this task, the laser was locked to the transmission peak of an external temperature stabilized $150 \mathrm{MHz}$ étalon (Burleigh CFT 500). Fine tuning of the laser is then easily done by varying the bias voltage on the $150 \mathrm{MHz}$ étalon until the molecular beam signal is maximized. The major source of creep off of the molecular beam signal is just the residual drift of the PZT of the $150 \mathrm{MHz}$ étalon. We have found that after an initial adjustment period of $1 / 2 \mathrm{~h}$, where the bias voltage of the $150 \mathrm{MHz}$ étalon is periodically adjusted to maximize the signal, the laser will sit within $10 \%$ of the signal maximum for times longer than $1 \mathrm{~h}$.

\section{The Ti:sapphire laser system}

The $0.8 \mu \mathrm{m}$ radiation is provided by a commercial Microlase (now Coherent/Scotland) MBR-110 Ti: $\mathrm{Al}_{2} \mathrm{O}_{3}$ laser system pumped by $5 \mathrm{~W}$ of $532 \mathrm{~nm}$ light from a Coherent Verdi Nd:Vanadate laser. The combination of a monolithic block resonator and the Verdi diode pump results in a laser system that is highly stable. When the MBR laser is locked to its internal reference cavity, the residual frequency jitter of the output is on the order of $70 \mathrm{kHz}$. As such, the lasers employed here required no additional stabilization.

Scanning of the $0.8 \mu \mathrm{m}$ laser is accomplished through use of its internal microprocessor controls, which allow continuous single mode scanning of up to $40 \mathrm{GHz}$. The scanning can either be regulated internally, or externally via a voltage ramp. For the experiments reported in this work, the laser scan was controlled by a voltage ramp generated by the controlling computer.

Because of the inherent stability of the MBR 110 , only a "slow" feedback loop was required to lock the BUC to the laser. In essence, the purpose of the loop is to correct for slow drifts in the laser frequency (such as when the laser is scanned) and voltage creep in the PZT of the BUC.

The Pound-Drever-Hall method, applied in transmission, was utilized to generate the error signal for the feedback loop. ${ }^{38}$ Approximately $800 \mathrm{~mW}$ of rf power at $\sim 1.7$ $\mathrm{MHz}$ was applied to an external electro-optic crystal (New Focus model 4002 broadband phase modulator) to generate sidebands. Both the carrier and sidebands were detected in transmission through the BUC using a high speed silicon photodiode (ThorLabs FDC 100). The photodiode signal was amplified and then mixed with the local oscillator to produce an error signal. The phase of the error signal was adjusted by passing it through two variable nanosecond delay boxes (Tennelec TC $412 \mathrm{~A}$ ) and then fed into a homemade servo circuit. The servo calculated proportional and integral parts, and then summed them to provide a corrective voltage for the PZT.

The corrective voltage and a computer generated voltage ramp were summed and used as input to a high voltage amplifier. The output from the amplifier was passed through a $1.2 \mathrm{~Hz}$ low pass filter and then applied to the piezo of the BUC. As the laser is scanned, the corrective voltage steadily increases. When the corrective voltage exceeds a preset threshold, the computer pauses the scan while it adjusts the voltage ramp to minimize the corrective voltage and then resumes the scan. By having everything under computer control, it is possible for the system to scan $40 \mathrm{GHz}$ (the maximum scanning range of the MBR laser) unattended, even though the free spectral range of the BUC is only $6 \mathrm{GHz}$. The computer merely resets the cavity voltage at the end the ramp, and relocks the BUC at the lower end of the voltage ramp.

Absolute frequency reference is provided by a homebuilt wave meter. ${ }^{39}$ Comparison of the wave meter readings with literature values for the $0 \rightarrow 4$ transition of acetylene reveal a systematic error of approximately $0.02 \mathrm{~cm}^{-1}$. This error is likely due to the dispersion of air, which is calculated to lead to a shift of about $0.017 \mathrm{~cm}^{-1}$ in this wavelength region. Relative frequency calibration is performed by the simultaneous monitoring of a hermetically sealed, temperature stabilized $750 \mathrm{MHz}$ confocal scanning étalon. The étalon is ramped, and the voltage that corresponds to the maximum of the transmitted fringe is provided by a sample-andhold type circuit. ${ }^{40}$ The residual drift of the étalon is on the order of $30 \mathrm{MHz} / \mathrm{h}$. 


\section{Computer system}

The entire experiment is digitally controlled by a Pentium III computer system (Gateway GP7-450). The computer is equipped with a 64 channel, high speed 12-bit analogdigital card (National Instruments PCI-6071E), and a six channel analog output card (National Instruments AT-A06). The cards are controlled by a program written in National Instrument's LABVIEW Instrument language. The program is responsible for confirming the lock of the $1.5 \mu \mathrm{m}$ laser, acquiring and maintaining the lock of the $0.8 \mu \mathrm{m}$ BUC, scanning the $0.8 \mu \mathrm{m}$ laser, recording the bolometer signal, and recording the signal from the $750 \mathrm{MHz}$ scanning étalon.

\section{RESULTS}

The first step in testing the instrument was to optimize the molecular beam signal itself. To accomplish this task, the beam was modulated at $\sim 30 \mathrm{~Hz}$ with a mechanical chopper, and the resulting bolometer signal recorded. For a pure $\mathrm{He}$ beam at a stagnation pressure of 8 bar, the helium beam signal was measured to be $115 \mathrm{mV}$. Based on this figure, and the previously mentioned molecular beam and bolometer parameters, a number of parameters governing the performance of the spectrometer can be calculated.

The $115 \mathrm{mV}$ signal corresponds to approximately 1 $\times 10^{14}$ atoms/s impacting upon the bolometer. With the dimensions of the machine, this corresponds to $8 \times 10^{17}$ atoms/( $\left.\mathrm{s}^{*} \mathrm{sr}\right)$. Since the concentration of a typical analyte molecule seeded in $\mathrm{He}$ is $1 \%$, there will be $1 \times 10^{12}$ analyte molecules/s incident upon the bolometer. However, not all the molecules that the bolometer sees will have been pumped by the lasers. The residual divergence of the portion of the molecular beam that hits the bolometer is $5.26 \mathrm{mrad}$, which means that the molecular beam size in the cavities is larger than the cavity beam waists. Based upon the residual beam divergence and the size of the cavity beam waists calculated earlier, only $30 \%$ of the analyte molecules are illuminated by the $1.5 \mu \mathrm{m}$ laser, and only $16 \%$ by the $0.8 \mu \mathrm{m}$ laser. Because the $0.8 \mu \mathrm{m}$ cavity beam waist is smaller than the $1.5 \mu \mathrm{m}$ beam waist, and the fact that the molecular beam expands as it travels from the 1.5 to the $0.8 \mu \mathrm{m}$ cavity, it is clear that any molecules pumped by the $0.8 \mu \mathrm{m}$ cavity have already been illuminated by $1.5 \mu \mathrm{m}$ radiation, if the two cavities are properly aligned with respect to each other. If we assume that the analyte molecules are in a single rotational state, a cw saturated $0 \rightarrow 2$ first step should prepare $8 \times 10^{10}$ excited molecules/s for further excitation to the $v=6$ manifold. Saturation of the subsequent $2 \rightarrow 6$ transition should yield 2 $\times 10^{10}$ excited molecules/s (a factor of 2 loss each for saturation and the duty cycle of the chopper).

Of course there are many rotational levels that are thermally populated, even at $3 \mathrm{~K}$. For HCN, roughly half of the molecules are in the $J=1$ level. Therefore, with a bolometer responsivity of $2.32 \times 10^{5} \mathrm{~V} / \mathrm{W}$, we expect a saturated $R(1)$ transition for the $0 \rightarrow 2$ step to yield a signal level of $\sim 1 \mathrm{mV}$. In order to label the double resonance signal, we will adopt a nomenclature that corresponds to the individual rotational transitions associated with each step, with the $0 \rightarrow 2$ step listed first. Therefore, an $R(1) R(2)$ transition corresponds to

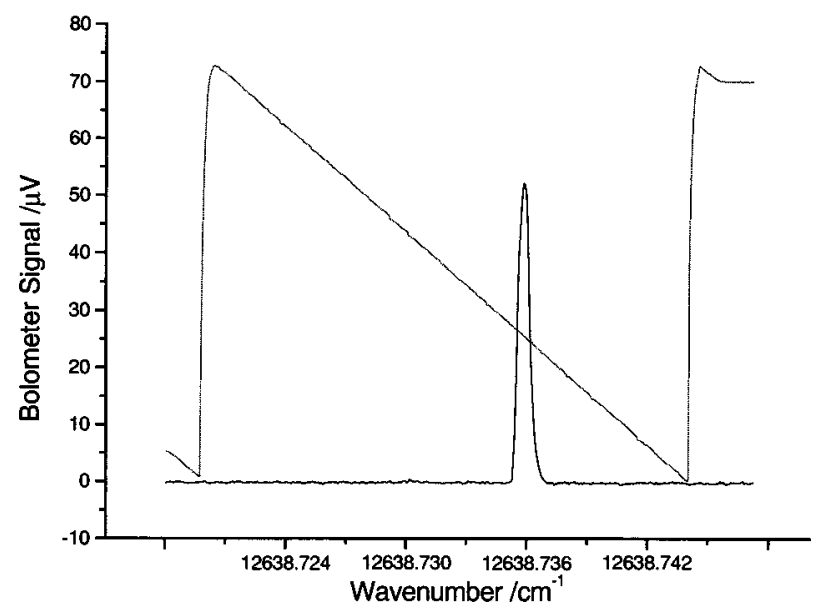

FIG. 3. Plot of the $R(1)$ transition in the $4 \nu_{3}$ band of HCN. The sawtooth line is the $750 \mathrm{MHz}$ scanning étalon.

an $R(1)$ transition for the $0 \rightarrow 2$ step and an $R(2)$ transition for the $2 \rightarrow 6$ step, which corresponds to an $S(1)$ transition if a single photon $0 \rightarrow 6$ transition was taking place. A saturated $R(1) R(2)$ transition for the $0 \rightarrow 2 \rightarrow 6$ transition is expected to give $\sim 500 \mu \mathrm{V}$ of signal (the photon energy for the $2 \rightarrow 6$ step is roughly twice as large as for the $0 \rightarrow 2$ step).

The aforementioned residual beam divergence corresponds to a residual Doppler broadening of $11 \mathrm{MHz}$. When summed in quadrature with the previously calculated transit time broadening of $5 \mathrm{MHz}$, this yields an expected linewidth of $12 \mathrm{MHz}$. In order to test the performance of the spectrometer, various spectra of known molecular transitions in $\mathrm{HCN}^{41,42}$ were recorded. Each step in the experiment $(0$ $\rightarrow 2$ and $0 \rightarrow 4$ ) was tested separately, and then finally they were combined to yield a $0 \rightarrow 2 \rightarrow 6$ spectrum.

\section{A. $0 \rightarrow 2$ transitions}

Testing and optimization of the $0 \rightarrow 2$ transition was done using the $2 \nu_{3}$ vibration of $\mathrm{HCN}$. We found that the 1.5 $\mu \mathrm{m}$ laser was powerful enough to saturate the transition, since attenuating the laser power by a factor of 5 did not produce any noticeable change in the signal. At 8 bar stagnation pressure, a typical bolometer signal for the $R(1)$ transition located at $6525.373 \mathrm{~cm}^{-1}$ was $300 \mu \mathrm{V}$. With the previously stated bolometer responsivity of $2.32 \times 10^{5} \mathrm{~V} / \mathrm{W}$, this corresponds to approximately $10^{10}$ molecules/s being excited. When the stagnation pressure was reduced to 3 bar, the $R$ (1) signal increased to $450 \mu \mathrm{V}$. This indicates that there is significant dimerization of HCN occurring at higher pressures. Fitting the other observed $R$-line intensities gives an estimate for the molecular beam temperature of $3 \mathrm{~K}$.

The observed signal for the $0 \rightarrow 2$ step is about a factor of three less than the calculated "ideal" value of the previous section. We attribute the discrepancy to dimerization of $\mathrm{HCN}$ and miscellaneous experimental nonidealities.

\section{B. $0 \rightarrow 4$ transitions}

The $4 \nu_{3}$ transition in HCN was used to optimize the Ti:sapphire BUC alignment. Figure 3 shows a spectrum of the $R(0)$ line of this transition at $12638.74 \mathrm{~cm}^{-1}$. The peak 


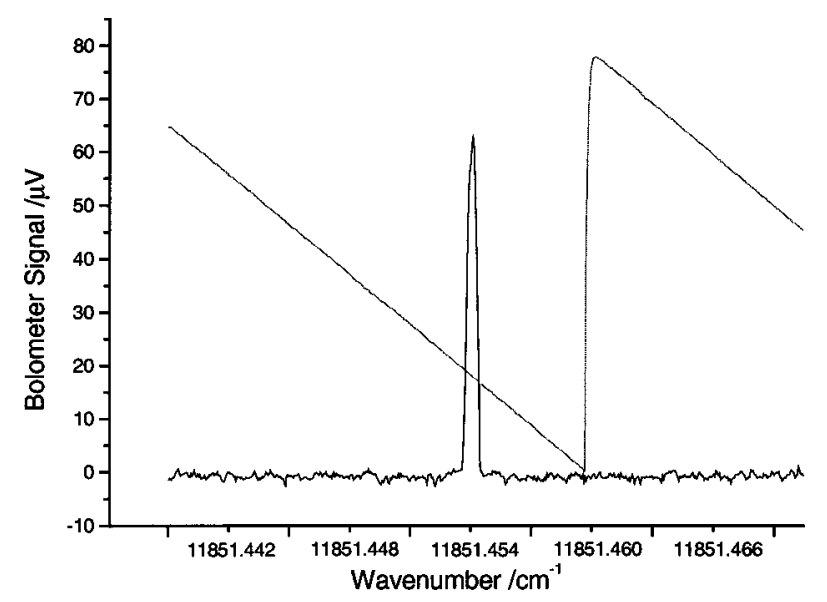

FIG. 4. Plot of the $R(1) R(2)$ transition in the 006 band of HCN. The photon energy of the scanned $(0.8 \mu \mathrm{m})$ laser is shown on the $x$ axis. The total photon energy absorbed by the molecules is $18376.83 \mathrm{~cm}^{-1}$.

height is slightly larger than $50 \mu \mathrm{V}$, which when coupled with the experimental noise of $25 \mathrm{nV}^{*} \mathrm{~Hz}^{-1 / 2} \mathrm{rms}$, corresponds to a signal-to-noise ratio of $2000 \mathrm{~Hz}^{-1 / 2}$. Based upon our bolometer responsivity, this corresponds to $9 \times 10^{8}$ molecules/s excited to the $v=4$ level. A power dependence of the spectrum showed linear behavior, which indicates that, as expected, we are far from saturating this transition. The observed linewidth is $15 \mathrm{MHz}$ (FWHM), in good agreement with the calculated linewidth of $12 \mathrm{MHz}$ based on the transit time broadening $(5 \mathrm{MHz})$ and residual Doppler broadening (11 $\mathrm{MHz}$ ).

\section{C. $0 \rightarrow 2 \rightarrow 6$ transitions}

Figure 4 shows a line from the 006 band in hydrogen cyanide - the first double resonance signal obtained in this spectrometer. The overall transition is an $R(1)$ transition in the $0 \rightarrow 2$ manifold followed by an $R(2)$ transition in the 2 $\rightarrow 6$. In accordance with the nomenclature we described above, this corresponds to an $R(1) R(2)$ transition. The total photon energy adsorbed by the molecule is $18376.83 \mathrm{~cm}^{-1}$ (the sum of the photon energies for each step). The peak height is $62 \mu \mathrm{V}$, which corresponds to $1.1 \times 10^{9}$ molecules/s excited to the $v=6$ level, and the noise level is approximately $80 \mathrm{nV}^{*} \mathrm{~Hz}^{-1 / 2} \mathrm{rms}$. The signal-to-noise ratio of the spectrum is therefore around $775 \mathrm{~Hz}^{-1 / 2}$ and the linewidth is the same as for the $0 \rightarrow 4$ transition.

Since the factor of two loss (for a saturated transition) in available molecules after the $0 \rightarrow 2$ step is offset by a photon energy for the $2 \rightarrow 6$ step that is twice as large, the signal level of the $2 \rightarrow 6$ step should be the same as the $0 \rightarrow 2$ step. Of course, this is only true if all the transitions are saturated and the $0.8 \mu \mathrm{m}$ laser pumps the same portion of the molecular beam as the $1.5 \mu \mathrm{m}$ laser. However, given that the 0.8 $\mu \mathrm{m}$ beamwaist is smaller than the $1.5 \mu \mathrm{m}$ beamwaist, even with "optimal" alignment, the $0.8 \mu \mathrm{m}$ cavity will only interact with about half the molecules that the $1.5 \mu \mathrm{m}$ cavity pumped, as calculated earlier. Since the observed double resonance signal is a factor of five lower than the $0 \rightarrow 2$ signal, it is clear that we are not saturating the $2 \rightarrow 6$ transition. This conclusion is further supported by Fig. 5, which

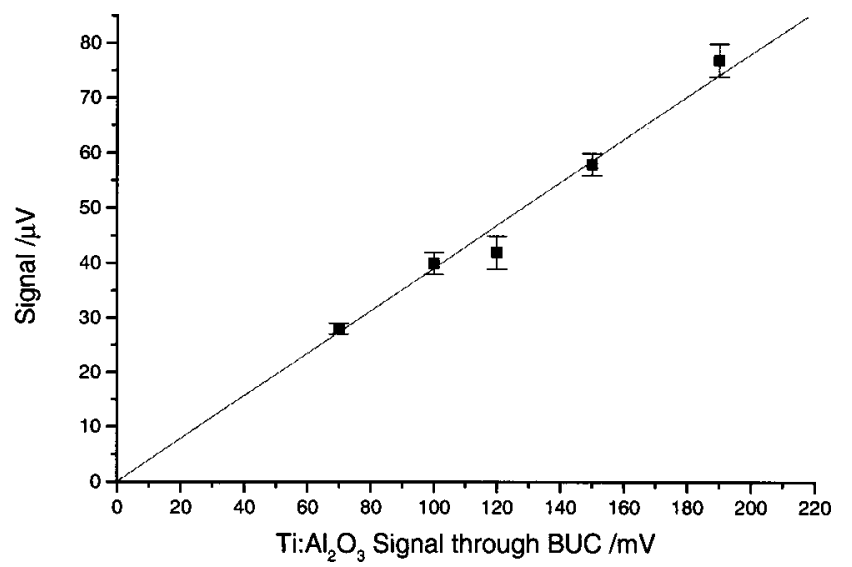

FIG. 5. Plot of the $2 \rightarrow 6$ signal intensity as a function of $\mathrm{Ti}: \mathrm{Al}_{2} \mathrm{O}_{3}$ laser power (measured by a photodiode in transmission through the BUC) for the transition shown in Fig. 4. The linearity of the plot indicates that we are not saturating the transition.

shows a plot of the bolometer signal as the power of the Ti:sapphire laser is systematically varied. As shown, the plot is very linear, which again indicates that we are far from the saturation regime of the $2 \rightarrow 6$ transition. From the fact that the $2 \rightarrow 6$ transition is unsaturated we can estimate the maximum gain of the $0.8 \mu \mathrm{m}$ cavity. Based on the transition dipole for HCN, the power needed to saturate the $2 \rightarrow 6$ transition is $45 \mathrm{~W}^{33} 100 \mathrm{~mW}$ of laser power was coupled into the optical fiber, and half of that is expected to couple into the cavity. Therefore, the gain of the cavity has to be less than 900 , in order for the circulating laser power to be less than $45 \mathrm{~W}$.

The higher noise present in Fig. 4 versus that of Fig. 3 is due to the fact that the signal is not background free-that is, the $2 \rightarrow 6$ transition sits on top of a large dc $0 \rightarrow 2$ transition. Minor frequency and amplitude fluctuations of the $0 \rightarrow 2$ laser cause large dc changes in the first step, and are responsible for the larger noise of the sequential double resonance signal. On the other hand, long term drift of the $150 \mathrm{MHz}$ étalon only results in a loss of signal, as there are less molecules in the $v=2$ level available to be pumped to the $v$ $=6$ level, and not an increase in noise. Long term drift, however, does mean that relative intensity information is not as reliable as in a single photon experiment. To avoid this problem, a more complex double modulation scheme could be adopted, and the signal from the $0 \rightarrow 2$ step used to keep the $150 \mathrm{MHz}$ étalon on top of the molecular beam resonance.

\section{ACKNOWLEDGMENTS}

The authors are grateful to Larry Darazio and Werner Schiedt for their help in constructing the apparatus, and to Paul Rabinowitz for the loan of a lock-in amplifier. This work was supported by the NSF.

\footnotetext{
${ }^{1}$ R. Mecke and R. Ziegler, Z. Phys. 101, 405 (1936).

${ }^{2}$ B. R. Henry, Acc. Chem. Res. 10, 207 (1977).

${ }^{3}$ M. S. Child and L. Halonen, Adv. Chem. Phys. 57, 1 (1984).

${ }^{4}$ N. B. Slater, Theory of Unimolecular Reactions (Cornell University Press, Ithaca, NY, 1959).

${ }^{5}$ R. A. Marcus and O. K. Rice, J. Phys. Colloid Chem. 55, 894 (1951); J. Chem. Phys. 20, 359 (1952).
} 
${ }^{6}$ M. Quack, Annu. Rev. Phys. Chem. 41, 839 (1990).

${ }^{7}$ D. J. Nesbitt and R. W. Field, J. Phys. Chem. 100, 12735 (1996).

${ }^{8}$ K. K. Lehmann, G. Scoles, and B. H. Pate, Annu. Rev. Phys. Chem. 45, 241 (1994)

${ }^{9}$ M. Gruebele and R. Bigwood, Int. Rev. Phys. Chem. 17, 91 (1998).

${ }^{10}$ A. McIlroy, D. J. Nesbitt, E. R. T. Kerstel, B. H. Pate, K. K. Lehmann, and G. Scoles, J. Chem. Phys. 100, 2596 (1994).

${ }^{11}$ U. Merker, H. K. Srivastava, A. Callegari, K. K. Lehmann, and G. Scoles, Phys. Chem. Chem. Phys. 1, 2427 (1999).

${ }^{12}$ J. W. Dolce, A. Callegari, B. Meyer, K. K. Lehmann, and G. Scoles, J. Phys. Chem. 107, 6549 (1997).

${ }^{13}$ A. Callegari, H. K. Srivastava, U. Merker, K. K. Lehmann, G. Scoles, and M. J. Davis, J. Chem. Phys. 106, 432 (1997).

${ }^{14}$ J. E. Gambogi, R. Z. Pearson, X. M. Yang, K. K. Lehmann, and G. Scoles, Chem. Phys. 190, 191 (1995).

${ }^{15}$ J. H. Timmermans, K. K. Lehmann, and G. Scoles, Chem. Phys. 190, 393 (1995).

${ }^{16}$ J. E. Gambogi, R. P. L'Esperance, K. K. Lehmann, and G. Scoles, J. Phys. Chem. 98, 5614 (1994).

${ }^{17}$ J. E. Gambogi, E. R. T. Kerstel, K. K. Lehmann, and G. Scoles, J. Chem. Phys. 100, 2612 (1994).

${ }^{18}$ D. Green, S. Hammond, J. Keske, and B. H. Pate, J. Chem. Phys. 110, 1979 (1999).

${ }^{19}$ D. A. McWhorter and B. H. Pate, J. Mol. Spectrosc. 193, 159 (1999).

${ }^{20}$ D. A. McWhorter and B. H. Pate, J. Phys. Chem. A 102, 8786 (1998).

${ }^{21}$ A. M. Andrews, G. T. Fraser, and B. H. Pate, J. Chem. Phys. 109, 4290 (1998).

${ }^{22}$ S. Cupp, C. Y. Lee, D. A. McWhorter, and B. H. Pate, J. Chem. Phys. 109, 4302 (1998).

${ }^{23}$ E. Hudspeth, D. A. McWhorter, and B. H. Pate, J. Chem. Phys. 109, 4316 (1998).

${ }^{24}$ D. Green, R. Holmberg, C. Y. Lee, D. A. McWhorter, and B. H. Pate, J. Chem. Phys. 109, 4407 (1998).
${ }^{25}$ J. Go and D. S. Perry, J. Chem. Phys. 103, 5194 (1995).

${ }^{26}$ D. S. Perry, G. A. Bethardy, and X. L. Wang, Ber. Bunsenges. Phys. Chem. 99, 530 (1995).

${ }^{27}$ G. A. Bethardy, X. L. Wang, and D. S. Perry, Can. J. Chem. 72, 652 (1994).

${ }^{28}$ J. S. Go, T. J. Cronin, and D. S. Perry, Chem. Phys. 175, 127 (1993).

${ }^{29}$ G. T. Fraser, B. H. Pate, G. A. Bethardy, and D. S. Perry, Chem. Phys. 175, 223 (1993).

${ }^{30}$ R. D. F. Settle and T. R. Rizzo, J. Chem. Phys. 95, 2823 (1992).

${ }^{31}$ O. V. Boyarkin, T. R. Rizzo, and D. S. Perry, J. Chem. Phys. 110, 11346 (1999); 110, 11359 (1999).

${ }^{32}$ O. V. Boyarkin, L. Lubich, R. D. F. Settle, D. S. Perry, and T. R. Rizzo, J. Chem. Phys. 107, 8409 (1997).

${ }^{33}$ J. E. Gambogi, Ph.D. thesis, Princeton University, 1995.

${ }^{34}$ The nozzle is constructed from a molybdenum electron microscopy disc aperture from Ted Pella Inc., mounted on a copper tube.

${ }^{35}$ W. Demtröder, Laser Spectroscopy (Springer, Berlin, 1996).

${ }^{36}$ A. Callegari, Ph.D. thesis, Princeton University, 1998.

${ }^{37}$ R. Beigang, G. Litfin, and H. Welling, Opt. Commun. 22, 269 (1977).

${ }^{38}$ R. W. P. Drever, J. L. Hall, F. V. Kowalski, J. Hough, G. M. Ford, A. J. Munley, and H. Ward, Appl. Phys. B: Photophys. Laser Chem. 31, 97 (1983).

${ }^{39}$ The wave meter was built by D. Ramanini based upon a design by $\mathrm{H}$. Lew, N. Marmet, M. D. Marshall, A. R. McKellar, and G. W. Nichols, Appl. Phys. B: Photophys. Laser Chem. 42, 5 (1987).

${ }^{40}$ This circuit was designed and built by W. S. Woodward, Digital Specialties, 1702 Allan Road, Chapel Hill, NC 27514.

${ }^{41}$ A. M. Smith, S. L. Coy, and W. Klemperer, J. Mol. Spectrosc. 134, 134 (1989).

${ }^{42}$ K. K. Lehmann, G. J. Scherer, and W. Klemperer, J. Chem. Phys. 77, 2853 (1982) 